\title{
12-PULSE RECTIFIER FOR TRACTION SUBSTATIONS OF ELECTRIC TRANSPORT
}

\author{
A. Poleshchuk \\ Novosibirsk State Technical University \\ Novosibirsk, Russian Federation \\ Nya999999@yandex.ru
}

\author{
M. Vilberger \\ Novosibirsk State Technical University \\ Novosibirsk, Russian Federation \\ vilberger@corp.nstu.ru
}

\begin{abstract}
The article discusses the influence of the nonsinusoidal form of the voltages of the supplying threephase network on the quality of the rectified voltage from multipulse rectifier with a circular outline of the valve shoulder. The research aims at identifying the most stable rectification circuit during the rectifier mains supply from the central electricity network.
\end{abstract}

Keywords-12-pulse rectifier; traction substations; electric vehicle; electromagnetic compatibility.

\section{INTRODUCTION}

Converters are used with a number of pulsations in the rectified voltage curve, greater than six on traction substations. Until recently, zero-pulse six-pulse rectification circuits "two back-to-back stars with an equalization reactor", a three-phase bridge were widely used. Rectifiers were also used with a cascade circuit for switching gates in the experimental order.

One of the most important directions of scientific and technical research is improving circuit AC to DC converters. The transition from classical rectification circuits to pulsed high-frequency rectifier-inverter converters solves some of the problems inherent in classical rectifier units, for example, the problem of improving the power factor. At the same time, rectifying circuits with multiphase converter transformers and rectifiers of electric currents of industrial frequency will be used in the transformation in industries with powerful and super-power consumers for a long time. Considering the prospects of using classical schemes, it is necessary to pay attention on the possibilities of increasing the energy efficiency of rectifiers built according to other schemes. Moreover, the improvement of the conversion quality, directly related to economy, can be achieved by circuit improvement of rectifier units.

Analysis of the existing schemes of the multi-pulse rectifying unit shows that the circuit design of the rectifier determines the value of the ratio of the secondary windings of the transformers in terms of power, that is, the weight and size parameters of the rectifiers [4]. A great deal of attention was paid to the improvement of methods for calculating installed capacities and energy parameters of gate converters in the works of the scientists of Novosibirsk State Technical
University, G.V. Grabovetsky, S.A. Kharitonov, G.S. Zinoviev, G.N. Vorfolomeev, N.I. Shchurov, S.V. Mjatezh.

A significant reduction in power losses during the conversion of alternating current to direct current can be expected if the existing rectifying units are converted (or when new rectifying units are installed) in accordance with schemes ensuring a higher pulse rate of the rectified voltage. At the same time, losses can be reduced not only in the rectifying unit itself, but also in the supply networks and adjacent consumers by reducing the distorting influence of the nonlinear load, which is a rectifying unit, on the qualitative characteristics of the networks. With increasing rectification of rectified voltage in DC networks, the requirements to masssize and cost parameters of smoothing filters of the traction network are reduced. In addition, power losses during filtration are also reduced [9]. However, studies of the electromagnetic compatibility of multi-pulse rectifiers and an assessment of their economics, even assuming symmetry and sinusoidal feeding of three-phase supply voltages performed in known works, do not allow one to fully evaluate their influence on the devices of signaling and communications, devices of electric rolling stock [5]

In connection with the above-mentioned problems, one can say that the development and research of multi-pulse rectifiers, combining the economics of conversion, simplicity and reliability of circuit designs, when operating under conditions of asymmetric and non-sinusoidal voltages of the supply network, are of great interest.

\section{WORK Of Twelve-Pulse RectifiERs With THE ACCOUNT OF ASYMMETRY AND NONSINUSOIDALITY OF NUTRIENT VOLTAGE}

Voltages generated at power plants are distorted due to the work of nonlinear loads (powerful electrotechnical installations, frequency-controlled drives, rectifiers, etc.) in the power system feeding multipulse rectifiers. Distortions of voltages affect the operation of the rectifier as a whole and they lead to distortion of the rectified voltage, which considerably reduces the conversion efficiency and requires additional costs [8].

Many Russian scientists have carried out research in the field of the influence of higher harmonics (which are present 
in the supply network), on the operation of rectifiers with a different number of pulsations of the rectified voltage at the converter output. They are B. Shlyaposhnikov, V. Posse, A. Matsenko, A. Ponomarev, A. Maslennikov, M. Treivas, O. Maglaperidze, Y. Anisimov, S. Sokolov, C. Chernikov, A. Pincov, M. Bader, A. Shidlovskiy, A. Nizov, M. Shalimov, V. Matsenko. In the works under consideration, almost no attention is paid to the rectifiers and their structures, which differ from the traditional design. In addition, these studies were aimed, as a rule, to investigate the influence of only one of the higher harmonics of the supply voltage, which is due in large part to the absence of a computer, which made it impossible to carry out a lot of calculations and adequately display the processes in the rectifier aggregates.

In this regard, the task was set to compile a mathematical model that allows calculating multipulse rectifiers and calculate the parameters characterizing the quality of the rectified voltage, taking into account the nonsinusoidality and unbalance of the supply voltage.

The quality of three-phase power supply systems of the central power supply is regulated by Russian national standard GOST 523144-2013. It shows the levels of the voltages harmonic composition for various points of common connection to electrical networks with different values of the nominal voltage. So one can conclude that the choice of the circuit solution of the twelve-pulse rectified voltage is directly related to the level of distortion of the mains voltage supplying this rectifier. Also the principle of the converter has an important role.

\section{A. The mathematical model of a three-phase supply network}

The coefficients of the n-th harmonic voltage component in percent are given in Russian national standard GOST 523144-2013 table. They are normally permissible for the corresponding points of common connection in networks with a nominal voltage of $10 \mathrm{kV}$. The maximum permissible values of the coefficients of the voltage harmonics are determined by the equation (1):

$$
K_{U(n)}=K_{D} \cdot K_{U(n) \text { norm }},
$$

$K_{D}$ is the coefficient of change in the percentage composition of higher harmonics, it has a change limit from 0 to 1.5. And at a value of $K_{D}=1.5$, the coefficients of the harmonic components will characterize the maximum permissible values, and for $\mathrm{K}_{\mathrm{D}}=1$, they are normally admissible.

Normal and maximum permissible values of asymmetry are regulated within the limits of 2 and $4 \%$ in Russian national standard GOST 523144-2013. The coefficient of asymmetry is calculated in accordance with equation (2):

$$
K_{U}=\frac{\left|U_{I I}\right|}{\left|U_{I}\right|} \cdot 100 \%
$$

$\mathrm{U}_{\mathrm{I}}-$ the module voltage phase sequence;

$\mathrm{U}_{\mathrm{II}}-$ the module voltage reverse phase sequence.
Complexes of currents of direct and reverse sequences are found from equations (3) and (4):

$$
\begin{aligned}
& \dot{U}_{I}=\frac{1}{3} \cdot\left(\dot{U}_{A}+a \cdot \dot{U}_{B}+a^{2} \cdot \dot{U}_{C}\right) ; \\
& \dot{U}_{I I}=\frac{1}{3} \cdot\left(\dot{U}_{A}+a^{2} \cdot \dot{U}_{B}+a \cdot \dot{U}_{C}\right),
\end{aligned}
$$

The determination of the stress values and their phase angles of displacement for a given asymmetry can be performed in accordance with the algorithm presented in Fig. 1. After setting the value of the level of one of the three-phase network voltages, the phase angle of the shift relative to the two other voltages is calculated under the condition that the sum of all the voltages is equal to zero, as shown in Fig. 2:

$$
\alpha=180-\frac{180}{\pi} \cdot \arccos \left(\frac{U_{A}}{2 \cdot U_{C}}\right),
$$

$\mathrm{U}_{\mathrm{A}}$ - the effective value of the phase A voltage, which is changed with respect to the other phase voltages;

$\mathrm{U}_{\mathrm{C}}=\mathrm{U}_{\mathrm{B}}$ - effective values of the voltage phases $\mathrm{B}, \mathrm{C}$.

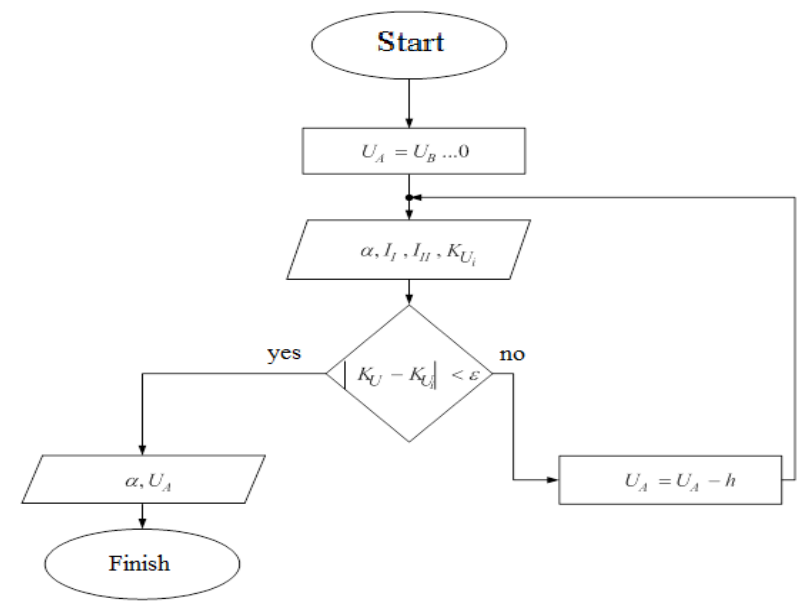

Fig. 1. - Algorithm for determining the level of voltage and phase angles of a three-phase voltage system.

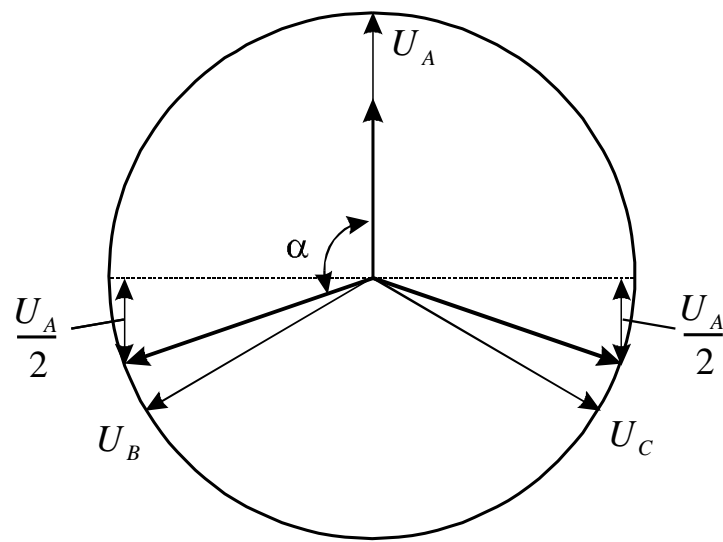

Fig. 2. - Explanation of the phase angle definition on a vector diagram 
After determining the phase angle according to expression (5), the asymmetry coefficient is determined according to formula (2) with the corresponding obtained values of the stresses. In the case when an asymmetry level is obtained, which was not specified initially, another voltage level is taken and the calculations are carried out until the set asymmetry value is obtained.

The voltages of the three-phase supply network, taking into account harmonics and asymmetry, are defined by the expressions:

$$
\begin{aligned}
& u_{A}=U_{A} \cdot\left[\sin (\omega t)+K_{\mathrm{D}} \cdot \sum_{i=2}^{\infty} K_{U \mathrm{norm}(\mathrm{i})} \cdot \sin (\omega t \cdot i)\right] \\
& u_{B}=U_{B} \cdot\left[\sin (\omega t)+K_{\mathrm{D}} \cdot \sum_{i=2}^{\infty} K_{U \mathrm{norm}(\mathrm{i})} \cdot \sin \left(\left(\omega t-\alpha_{B}\right) \cdot i\right)\right] \\
& u_{C}=U_{C} \cdot\left[\sin (\omega t)+K_{\mathrm{D}} \cdot \sum_{i=2}^{\infty} K_{U \mathrm{norm}(\mathrm{i})} \cdot \sin \left(\left(\omega t+\alpha_{C}\right) \cdot i\right)\right]
\end{aligned}
$$

Calculations of the operation of the rectifiers can be performed based on the obtained expressions $(2,6)$ and the developed algorithm (Fig. 1), if they are powered by an asymmetrical and nonsinusoidal power source.

\section{ANALYSIS OF RECTIFIED Voltage TAKING INTO ACCOUNT} THE ASYMMETRY AND NONSINUSOIDALITY OF THE SUPPLY Network Of A TwElve-Pulse RectifiER With A RING

\section{CONSTRUCTION Of THE VALVE ARMS}

Rectifiers in systems of traction power supply are used to convert $\mathrm{AC}$ to $\mathrm{DC}$ on traction substations of mainline railways, metro and city electric transport $[1,6]$.

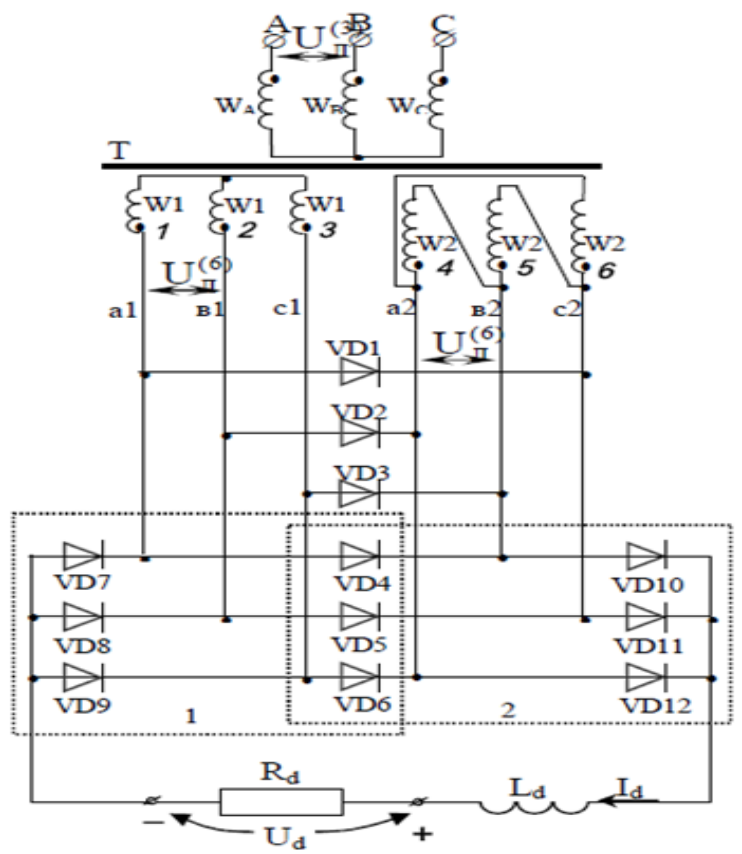

Fig. 3. - Schematic diagram of the ring rectifier
As a gate diagram, one can use a circular scheme for the construction of the valve shoulders developed in Novosibirsk State Technical University (Fig. 3).

A ring rectifier (Fig. 3) includes three-phase transformer $\mathrm{T}$ and twelve valves VD1 - VD12, one primary and two secondary windings, which form the connections in a star and a triangle, placed on the terminals of the transformer [2]. The ends of the secondary windings (connected to the star) are connected to the lines, connecting the diodes of the anode group with the diodes in the chains in the middle [8].

The conclusions of the secondary windings of the transformer are connected to the lines connecting the middle diodes with cathode-group diodes that are triangle-connected. And if one side of the diode of the middle group is connected to the output of the phase of a star of one name, then on its other side is connected to the output of the phase of the triangle of the next denomination. The output of each of the phases of the star is connected through an additional diode with that phase of the triangle with which it is not connected by the diode of the bridge. Middle bridge diodes and additional diodes VD1 - VD3 form a closed ring of six diodes connected to each other only by the same named electrodes [3].

Thus, the reduction in electric energy losses due to the reduction in the number of diodes in the current flow circuit is obvious.

However, it is necessary to take into account that the power supply of rectifying units is realized in real conditions (in view of the presence of other nonlinear devices introducing their distortions into the voltage of the supplying three-phase network) by a three-phase asymmetric and non-sinusoidal voltage system.

Such distortion of the network affects, in turn, the operation of the rectifier as a whole and can reduce electromagnetic compatibility and its technical and economic performance. A mathematical model was developed to calculate the rectified voltage taking into account the characteristics of the supply network to determine the degree of influence of the distortion of the supply network voltage on the shape of the rectified voltage.

The rectified voltage at the output of the rectifying unit, made according to the scheme with the circular construction of the valve arms (Fig. 3), can be defined as the sum of the voltages:

$$
u_{d 1}=\left\{\begin{array}{l}
\left|u_{A \Delta} \cdot W_{1}-u_{B \Delta} \cdot W_{1}\right| \text { if } \\
\left|u_{A \Delta} \cdot W_{1}-u_{B \Delta} \cdot W_{1}\right|>\left|u_{A \Delta} \cdot W_{1}-u_{C \Delta} \cdot W_{1}\right| \wedge\left|u_{A \Delta} \cdot W_{1}-u_{B \Delta} \cdot W_{1}\right|>\left|u_{C \Delta} \cdot W_{1}-u_{B \Delta} \cdot W_{1}\right| \\
\left|u_{A \Delta} \cdot W_{1}-u_{C \Delta} \cdot W_{1}\right| \text { if } \\
\left|u_{A \Delta} \cdot W_{1}-u_{C \Delta} \cdot W_{1}\right|>\left|u_{A \Delta} \cdot W_{1}-u_{B \Delta} \cdot W_{1}\right| \wedge\left|u_{A \Delta} \cdot W_{1}-u_{B \Delta} \cdot W_{1}\right|>\left|u_{C \Delta} \cdot W_{1}+u_{B \Delta} \cdot W_{1}\right| \\
\left|u_{C \Delta} \cdot W_{1}-u_{B \Delta} \cdot W_{1}\right| \text { if } \\
\left|u_{C \Delta} \cdot W_{1}-u_{B \Delta} \cdot W_{1}\right|>\left|u_{A \Delta} \cdot W_{1}-u_{C \Delta} \cdot W_{1}\right| \wedge\left|u_{C \Delta} \cdot W_{1}-u_{B \Delta} \cdot W_{1}\right|>\left|u_{A \Delta} \cdot W_{1}+u_{B \Delta} \cdot W_{1}\right|
\end{array}\right.
$$




$$
u_{d 1}=\left\{\begin{array}{l}
\left|u_{A} \cdot W_{1}-u_{B} \cdot W_{1}\right| \text { if } \\
\left|u_{A} \cdot W_{1}-u_{B} \cdot W_{1}\right|>\left|u_{A} \cdot W_{1}-u_{C} \cdot W_{1}\right| \wedge\left|u_{A} \cdot W_{1}-u_{B} \cdot W_{1}\right|>\left|u_{C} \cdot W_{1}-u_{B} \cdot W_{1}\right| \\
\left|u_{A} \cdot W_{1}-u_{C} \cdot W_{1}\right| \text { if } \\
\left|u_{A} \cdot W_{1}-u_{C} \cdot W_{1}\right|>\left|u_{A} \cdot W_{1}-u_{B} \cdot W_{1}\right| \wedge\left|u_{A} \cdot W_{1}-u_{B} \cdot W_{1}\right|>\left|u_{C} \cdot W_{1}+u_{B} \cdot W_{1}\right| \\
\left|u_{C} \cdot W_{1}-u_{B} \cdot W_{1}\right| \text { if } \\
\left|u_{C} \cdot W_{1}-u_{B} \cdot W_{1}\right|>\left|u_{A} \cdot W_{1}-u_{C} \cdot W_{1}\right| \wedge\left|u_{C} \cdot W_{1}-u_{B A} \cdot W_{1}\right|>\left|u_{A} \cdot W_{1}+u_{B} \cdot W_{1}\right|
\end{array}\right.
$$

$$
u_{d}=u_{d 1}+u_{d 2}
$$

The set of expressions (7) - (9) is a mathematical model for calculating the instantaneous values of the rectified voltage as a function of the supply voltage parameters (including asymmetry and nonsinusoidality) [10]. This mathematical model was implemented in Mathcad and the implementation of the rectified voltage variation graphs under various conditions of influence on the rectification process of nonsinusoidal and asymmetry of the feeding three-phase network obtained, some of them are presented in Fig. 3 and Fig. 5.

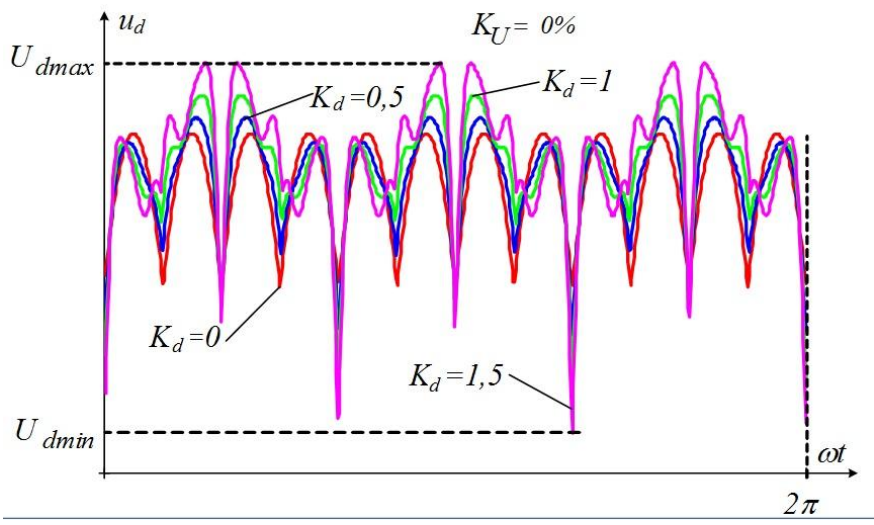

Fig. 4. - The rectified voltage of a 12-pulse rectifier at different coefficients $\mathrm{K}_{\mathrm{d}}$

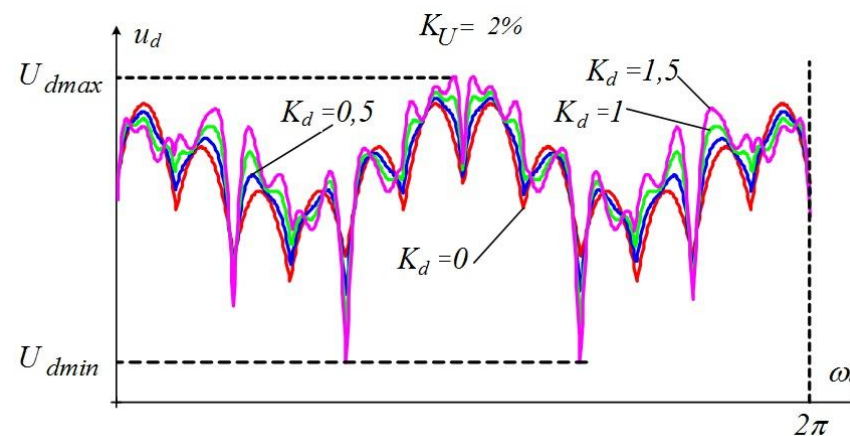

Fig. 5. - The rectified voltage of a 12-pulse rectifier with unbalance of the supply voltage of $2 \%$ and different coefficients $\mathrm{K}_{\mathrm{d}}$
TABLE I. THE RANGE OF PULSATIONS, $\Delta, \%$

\begin{tabular}{|l|l|l|l|l|l|l|l|l|}
\hline \multicolumn{7}{|c|}{$K_{U}=\%$} \\
\hline $\mathrm{K}_{\mathrm{d}}$ & 0.6 & 0.7 & 0.8 & 0.9 & 1.0 & 1.1 & 1.2 & 1.3 \\
\hline $\begin{array}{l}m q \\
12\end{array}$ & 5.2 & 5.5 & 5.8 & 6.1 & 6.5 & 6.9 & 7.2 & 7.5 \\
\hline $\mathrm{K}_{\mathrm{d}}$ & 0.6 & 0.7 & 0.8 & 0.9 & 1.0 & 1.1 & 1.2 & 1.3 \\
\hline $\begin{array}{l}m q \\
12\end{array}$ & 8.2 & 8.5 & 8.8 & 9.1 & 9.5 & 9.8 & 10.2 & 10.6 \\
\hline
\end{tabular}

The mathematical model was established for calculation of the rectified voltage based on the characteristics of the supply network with the purpose of identifying the degree of influence of the mains voltage distortion on the shape of the rectified voltage.

The straightening process can be divided into two stages of rectification to determine the instantaneous values of the rectified voltage of the rectifier under consideration. And then the rectified voltage of the entire transformer will be determined as the sum of rectified voltages, each of the stages, shifted by the corresponding phase angle.

\section{COMPARATIVE ANALYSIS OF THE RECTIFIED VOLTAGE QUALITY AT ASYMMETRY AND NON-SINUSOIDAL VOLTAGE OF THE SUPPLY NETWORK OF VARIOUS CIRCUIT SOLUTIONS}

The coefficient, defined as the ratio of the range of the ripple to the average value of the rectified voltage, is taken as one of the indices of the rectified voltage quality [7]:

$$
\Delta \%=\frac{U_{d \max }-U_{d \min }}{\frac{1}{2 \cdot \pi} \int_{0}^{2 \cdot \pi} u_{d 0}(\omega t) d \omega t} \cdot 100 \%
$$

$U_{d \max }, U_{d \min }$ - the maximum and minimum value of the rectified voltage.

The mathematical model in Mathsad for determining the amplitude of pulsations for various circuit solutions of twelvepulse rectifiers, taking into account the asymmetry and nonsinusoidal voltages of the supply network, is designed to estimate and to calculate the influence of nonsinusoidal and asymmetry supply voltages on the quality of the rectified voltage. The results of the solutions are shown in Fig. 6-7. 


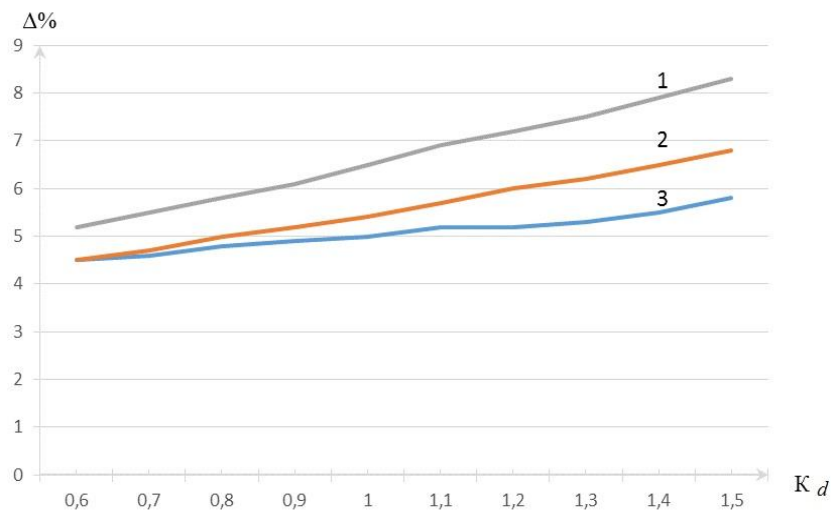

Fig. 6. - Graphical dependences of the pulsations range $\Delta \%$ when $\mathrm{K}_{\mathrm{U}}=0 \%$ on the coefficient of change in the percentage composition of higher harmonics for different circuit solutions of rectifiers: 1 - rectifier with ring construction of the valve arms, 2 - rectifier with series connection of threephase bridges, 3 - rectifier with orthogonal system of voltages.

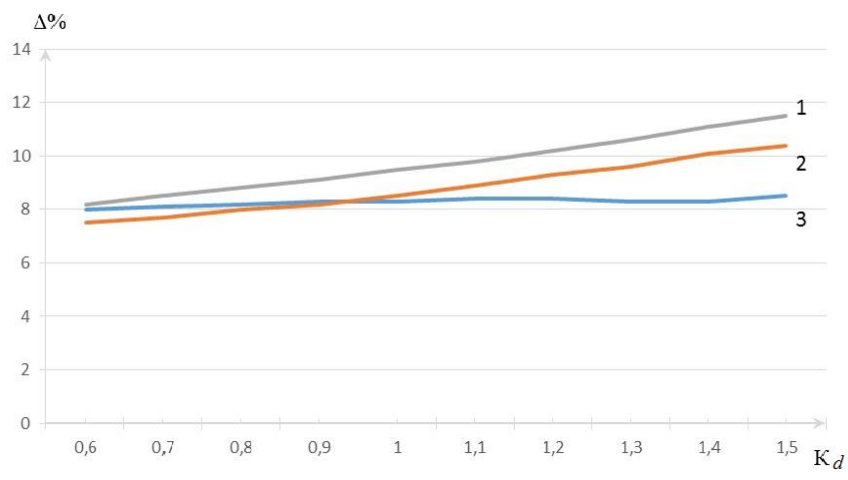

Fig. 7. - Graphical dependences of the pulsations range $\Delta \%$ when $\mathrm{K}_{\mathrm{U}}=2 \%$ on the coefficient of change in the percentage composition of higher harmonics for different circuit solutions of rectifiers: 1 - rectifier with ring construction of the valve arms, 2 - rectifier with series connection of threephase bridges, 3 - rectifier with orthogonal system of voltages.

As can be seen from Fig. 6-7, the rectified voltage at asymmetry of supply voltages has a larger pulse ripple than a symmetrical three-phase supply, which indicates a deterioration in the quality of the rectified voltage received. The circuit solution of a rectifier with an orthogonal voltage system is least influenced by the asymmetry and nonsinusoidal nature of the supply voltage, in comparison with a rectifier with a serial connection of the three-phase bridges and a rectifier with a ring construction of the valve arms.

\section{CONCLUSION}

A mathematical model has been established, it allows us to calculate the instantaneous value of the rectified voltage, depending on the non-sinusoidal mains voltage.

The mathematical model was implemented in MathCad and implementation schedules were obtained by rectified voltage changes at different levels of the fifth harmonic in the form of voltage curve.
It was found that the quality of the rectified voltage twelve-pulse rectifier is reduced by increasing the fifth harmonic in the mains voltage waveform. At the same time, as shown by research of the mathematical model, the quality of the rectified voltage is almost not affected by the harmonics multiple of three.

A technique for accounting for nonsinusoidality and asymmetry of the feeding three-phase network in calculating the multipulse rectifiers of orthogonal voltage systems was developed

The researches of rectifiers during operation in conditions of nonsinusoidal and unbalanced voltages of the supply network were made based on the developed mathematical model describing the operation of converters of stress systems with a 12-fold pulsation of rectified voltage. The most preferred rectification scheme, with the best quality of the rectified voltage, was a twelve-pulse rectifier circuit based on a three-phase transformer.

\section{References}

[1] V.I. Sopov, N.I. Schurov, Power supply systems electric vehicles DC, Novosibirsk: Publishing House of Novosibirsk State Technical University, 2013, pp. 700-728.

[2] S.A. Evdokimov, Structural synthesis of multi-phase rectifie transformers, monograph, Novosibirsk: Publishing House of Novosibirsk State Technical University, 2010, 423 p.

[3] E.A. Barinov, D.M. Strelnikova, M.E. Vilberger, A.V. Myatezh, "Work of 12-pulse rectifiers based on asymmetry and non-sinusoidal supply voltages," Electro. Electrical power industry, electrical industry, vol. 1, 68 p, 2014.

[4] S.V. Minezh, G.N. Vorolomeev, N.I. Shchurov, I.A. Tsiolina, S.A Evdokimov, "Technique for determining the installed powers of transformer converters of the number of phases", Proc. of Perfection of technical means of electric transport: Sat . Sci. Tr., pp. 21-28, 2002 [Sci. conference Perfection of technical means of electric transport, 2002].

[5] GN Vorolomeev, Methods and means of converting the number of phases to improve electromagnetic compatibility in electrical systems, Abstract of thesis. Dis. Dr. techn. Sciences, Novosib State. Tech. Un-t, Novosibirsk: Publishing house of NSTU, 1998, $41 \mathrm{p}$

[6] G. N. Vorolomeev, "Methods and means of converting the number of phases to improve electromagnetic compatibility in electrical systems", Abstract of thesis Dis. Dr. techn. Sciences: Novosibirsk: Publishing House of Novosibirsk State Technical University, 1998, 41 p

[7] G.N. Vorolomeev, S.A. Evdokimov, T.A. Rukosueva, M.E. Vilberger, EA Spiridonov, "Installed capacities of C-series rectifiers", Electrical engineering, electromechanics and electrotechnologies, materials of the second scientist-technical conference with international participation, pp. 141-144, October, 2005 [Second scientist-technical conference with international participation Electrical engineering, electromechanics and electrotechnologies, materials, 2005].

[8] N. N. Lopatkin, "Three-level rectifiers of three-phase orthogonal system of voltages", 8 International conference on actual problems of electronic instrument engineering: proceedings. APEIE, 2006

[9] D. Wojciechowski, Novel estimator of distorted and unbalanced electromotive force of the grid for control system of PWM rectifier with active filtering, EPE, 2005.

[10] P. Mysiak, "Multi-pulse diode rectifier with a coupled three-phase reactor - the design method and results of the simulation and laboratory tests”, Electrical Power Quality and Utilisation Journal, XI(1), 2005. 